\title{
Evaluation de la quantité des métaux lourds dans la décharge d'Agoè (Lomé- Togo) : cas du plomb, cadmium, cuivre, nickel et zinc
}

\author{
Magnoudéwa B. BODJONA ${ }^{1 *}$, Koffi A. KILI ${ }^{1}$, Sanonka TCHEGUENI ${ }^{1}$, \\ Bouchra KENNOU ${ }^{2}$, Gado TCHANGBEDJI ${ }^{1}$ et Mohamed El MERAY ${ }^{2}$ \\ ${ }^{1}$ Laboratoire GTVD (Gestion, Traitement et Valorisation des Déchets), Faculté des Sciences, \\ Université de Lomé, B.P. 1515, Lomé, Togo. \\ ${ }^{2}$ Laboratoire Physico-Chimie des Matériaux et Environnement (LPCME), Université Cadi Ayyad Boulevard le \\ Prince Moulay Abdallah, B.P.2390-40000, Marrakech, Maroc. \\ *Auteur correspondant, E-mail : bodjonabenoit@yahoo.fr; Tél : (00228) $22364160 / 90125411$
}

\section{RESUME}

Depuis l'indépendance (1960), la population de la ville de Lomé à l'instar des grandes villes Africaines n'a cessé d'augmenter, engendrant ainsi une forte production des déchets. Ceci a entraîné la mise en place des décharges à l'exemple de celle d'Agoè pour une gestion pérenne des déchets. Ce travail a pour objectif d'évaluer la quantité des métaux lourds de la décharge finale des déchets urbains de la ville de Lomé. Les échantillons sont prélevés à la surface et à chaque mètre en profondeur en six points de la décharge finale. Les paramètres physico-chimiques (humidité, $\mathrm{pH}$, matière organique) ont été déterminés sur ces échantillons de même que les teneurs de cinq métaux lourds (plomb, cadmium, nickel, cuivre et zinc). Le taux d'humidité des échantillons varie entre 17,65 et $67,64 \%$. Le $\mathrm{pH}$ mesuré varie entre 7,48 et 8,45. La teneur en matière organique déterminée par la méthode de perte au feu varie entre 8,58 et $14,57 \%$. Les teneurs moyennes des métaux lourds sont de $66,91 \mathrm{mg} / \mathrm{kg}$ pour le plomb; $2,51 \mathrm{mg} / \mathrm{kg}$ pour le cadmium; $6,10 \mathrm{mg} / \mathrm{kg}$ pour le nickel ; 70,24 mg/kg pour le cuivre et $401,13 \mathrm{mg} / \mathrm{kg}$ pour le zinc. Le cadmium et le zinc ont des teneurs supérieures à la norme AFNOR NF U 44-041.

(C) 2012 International Formulae Group. All rights reserved.

Mots clés : Déchets, décharge, métaux lourds, polluants.

\section{INTRODUCTION}

Les accroissements démographiques, économiques et urbains sont souvent à l'origine d'une grande production des déchets dans les grandes villes Africaines (Mokhtaria et al., 2007). Les déchets solides mis en décharge ne sont que très rarement inertes. De nombreuses réactions physico-chimiques et biologiques interviennent non seulement entre les déchets et le milieu récepteur (sol, substratum géologique, nappes, ...), mais également au sein même de ces déchets (Sbaa et al., 2001).

Les solutions d'élimination de ces déchets se limitent très souvent à leur enfouissement ou au remblayage des basfonds aux périphéries des villes (Koledzi et al., 2011). Les recherches ont montré que ces décharges publiques ont un impact négatif sur l'environnement, surtout avec la présence des métaux lourds (Mokhtaria et al., 2007). La composition chimique de ces déchets révèle 
des concentrations élevées en métaux lourds (Sbaa et al., 2001). Aussi, la pollution des eaux souterraines par les métaux lourds au voisinage des décharges a été souvent signalée dans la littérature (Chofqi et al., 2004). De nos jours, les problèmes causés par les métaux lourds sont devenus de plus en plus préoccupants, ils sont pour la plupart très dangereux lorsque ceux-ci parviennent à contaminer les eaux de boisson (M'leyeh et al., 2002). L'accumulation des métaux lourds dans l'environnement peut se répercuter sur la santé des êtres humains et des animaux (Wang et al., 2003). A l'échelle microscopique, les métaux ont aussi des effets néfastes sur les populations bactériennes, ce qui n'est pas sans conséquence sur le fonctionnement de l'écosystème.

Ainsi, l'évaluation des quantités des métaux lourds dans les déchets enfouis à la décharge est nécessaire et cela permettra d'évaluer leur potentiel polluant et, par conséquent d'entreprendre les mesures appropriées pour atténuer leur impact sur la santé des populations et sur l'environnement. La décharge finale de la ville de Lomé est une ancienne carrière qui se situe au Nord-Est de la ville dans le quartier Agoenyivé à environ 30 mètres de la nationale $\mathrm{N}^{\circ} 1$. Elle est la seule décharge finale de la ville. Le site géré par l'entreprise ICON SARL a une superficie de 6 ha environ et une profondeur moyenne de 6 mètres.

Ce travail a consisté à déterminer les éléments métalliques de la décharge finale de la ville de Lomé notamment, le plomb, le cadmium, le nickel, le cuivre et le zinc. Certains paramètres physico-chimiques comme l'humidité, le $\mathrm{pH}$ et la matière organique ont été également déterminés.

\section{MATERIEL ET METHODES Prélèvement des échantillons}

Les échantillons sont prélevés dans le mois de juillet en pleine saison humide. Ces prélèvements ont été effectués en six (6) points différents de la décharge. Ces points de prélèvement sont nommés puits et notés Pi (i caractérisant les points de prélèvement). L'âge d'enfouissement des déchets au niveau des points de prélèvement ou puits, varie entre 1 et 3 ans. Les prélèvements des déchets en profondeur sont faits à l'aide d'un camion à forage muni des tuyaux en acier de $100 \mathrm{~mm}$ de diamètre. Les échantillons en profondeur sont constitués à chaque mètre de profondeur jusqu'à atteindre la terre ferme, déterminant ainsi le fond de la décharge. Les profondeurs maximales sont de $4 \mathrm{~m}$ pour les puits P1 et $\mathrm{P} 2$; $5 \mathrm{~m}$ pour les puits $\mathrm{P} 3$ et $\mathrm{P} 6$; $6 \mathrm{~m}$ pour le puits P4 et $7 \mathrm{~m}$ pour le puits P5. Au laboratoire, les échantillons sont tamisés avec un tamis de $10 \mathrm{~mm}$ de diamètre, séchés et broyés afin d'obtenir des poudres bien homogènes.

\section{Analyses physico-chimiques \\ Humidité}

L'humidité a été déterminée par la méthode normée AFNOR NF U 44-171, qui consiste à une prise de masse de $100 \mathrm{~g}$ d'échantillon, mise à l'étuve à $105{ }^{\circ} \mathrm{C}$ jusqu'au poids constant (Charnay, 2005; Garcia et al., 2005; Yobouet et al., 2010). L'étuve utilisée est de type ISUZU Drying oven.

pH

Le $\mathrm{pH}$ a été déterminé dans une suspension de $10 \mathrm{~g}$ de déchet dans $25 \mathrm{ml}$ d'eau distillée. Après 30 minutes d'agitation à l'aide d'un agitateur magnétique, la suspension est laissée au repos pendant deux (2) heures et le pH est ensuite mesuré avec un pH-mètre à électrode de type CRISON pH 25. Matière organique

La teneur en matière organique est déterminée par calcination de la matière à 550 ${ }^{\circ} \mathrm{C}$ (Charnay, 2005; Alouéimine, 2006). Une masse de $10 \mathrm{~g}$ est calcinée à $550{ }^{\circ} \mathrm{C}$ pendant 4 heures au four électrique de type Nabertherm contrôleur P 320.

\section{Fluorescence $X$}

La fluorescence $X$ a permis de déterminer la composition chimique des échantillons de déchets. L'appareil utilisé est le spectromètre de fluorescence X 4 Pioneer. 


\section{Métaux lourds}

Les teneurs des métaux lourds totaux sont déterminées au spectromètre d'Emission Atomique-Plasma Couplé d'Induction (ICPAES) après minéralisation par attaque acide à chaud des échantillons à l'eau régale $(1 / 3 \mathrm{de}$ $\mathrm{HNO}_{3}$ et $2 / 3$ de $\mathrm{HCl}$ ).

\section{Traitements statistiques}

La valeur moyenne de chaque traitement, ainsi que la variance et l'écart-type correspondants, ont été calculés en tenant compte des trois répétitions réalisées. Les données ainsi obtenues ont fait l'objet d'analyse statistique avec le logiciel Assistat Version 7.6 Bêta (2012). L'analyse des variances (ANOVA) a été réalisée. Dans les cas où des différences significatives ont été trouvées $(\mathrm{p}<0,05)$, les moyennes ont été séparées en utilisant le test de Tukey ( $\mathrm{p}<$ $0,05)$.

\section{RESULTATS}

L'humidité d'un échantillon de déchets représente le rapport entre la masse d'eau présente dans cet échantillon et sa masse sèche. L'eau étant non seulement indispensable aux réactions biochimiques, elle permet aussi l'échange de nutriments et de microorganismes. L'humidité est donc nécessaire pour la dégradation des déchets. Les échantillons bruts, c'est-à-dire non tamisés, ont été utilisés pour la détermination du taux d'humidité. L'humidité des déchets enfouis à la décharge varie entre 17,65 et $67,64 \%$ (Figure 1). L'évolution de cette humidité en profondeur de la décharge n'est pas uniforme.

Le $\mathrm{pH}$ constitue un facteur dont le rôle est crucial pour la mobilité des ions métalliques, car il influence le nombre de charges négatives pouvant être mises en solution (McLaughlin et al., 2000). En effet, il existe un $\mathrm{pH}$ en dessous duquel les métaux sont brusquement relargués et il est évident que cela entraîne une remobilisation de ces métaux (Citeau, 2004). Le pH mesuré dans les échantillons varie entre 7,48 et 8,97 (Figure 2). Ces pH présentent un caractère basique.

La matière organique désigne les différents types de composés carbonés et azotés. La matière organique d'un déchet provient de plusieurs types de catégories de déchets, que sont les fermentescibles, les composés dégradés, les papiers cartons et les combustibles non classés (CNC) (Koledzi et al., 2011). Toutefois, d'autres composés difficilement dégradables tels que les plastiques représentent des sources importantes en matière organique. La matière organique est déterminée sur la fraction fine (déchets inférieurs à $10 \mathrm{~mm}$ ) des échantillons de déchets. La moyenne en matière organique de chaque puits varie entre 8,58 et $14,57 \%$ (Figure 3).

La fluorescence $\mathrm{X}$ est une analyse à la fois qualitative et quantitative. Elle a permis de déterminer la composition chimique des échantillons de déchets. Seuls les éléments majeurs sont présentés sur la Figure 4. Les résultats montrent la présence du chlore, des oxydes de silicium, d'aluminium, de fer, de titane, de manganèse, de sodium, de potassium, de calcium, du soufre et du potassium. Nous remarquons une forte présence de l'oxyde de silicium avec des teneurs qui sont autour de $50 \%$ dans tous les puits.

La pollution métallique des déchets enfouis est un problème à long terme, qui suscite beaucoup d'inquiétudes concernant leur relargage. Nous avons déterminé dans les échantillons de déchets enfouis à la décharge, les teneurs en matière sèche de cinq (5) métaux lourds les plus cités en littérature $(\mathrm{Pb}$, $\mathrm{Cd}, \mathrm{Ni}, \mathrm{Cu}$ et $\mathrm{Zn}$ ). Les résultats montrent que les teneurs moyennes dans les différents puits varient entre 19,81 et $159,66 \mathrm{mg} / \mathrm{kg}$ pour le plomb (Figure 5i) ; 1,32 et $3,81 \mathrm{mg} / \mathrm{kg}$ pour le cadmium (Figure $5 \mathrm{j}$ ) ; 3,25 et $13,48 \mathrm{mg} / \mathrm{kg}$ pour le nickel (Figure 5k); 44,99 et 94,27 $\mathrm{mg} / \mathrm{kg}$ pour le cuivre (Figure $5 \ell$ ) ; 216,51 et $507,81 \mathrm{mg} / \mathrm{kg}$ pour le zinc (Figure $5 \mathrm{~m}$ ). 


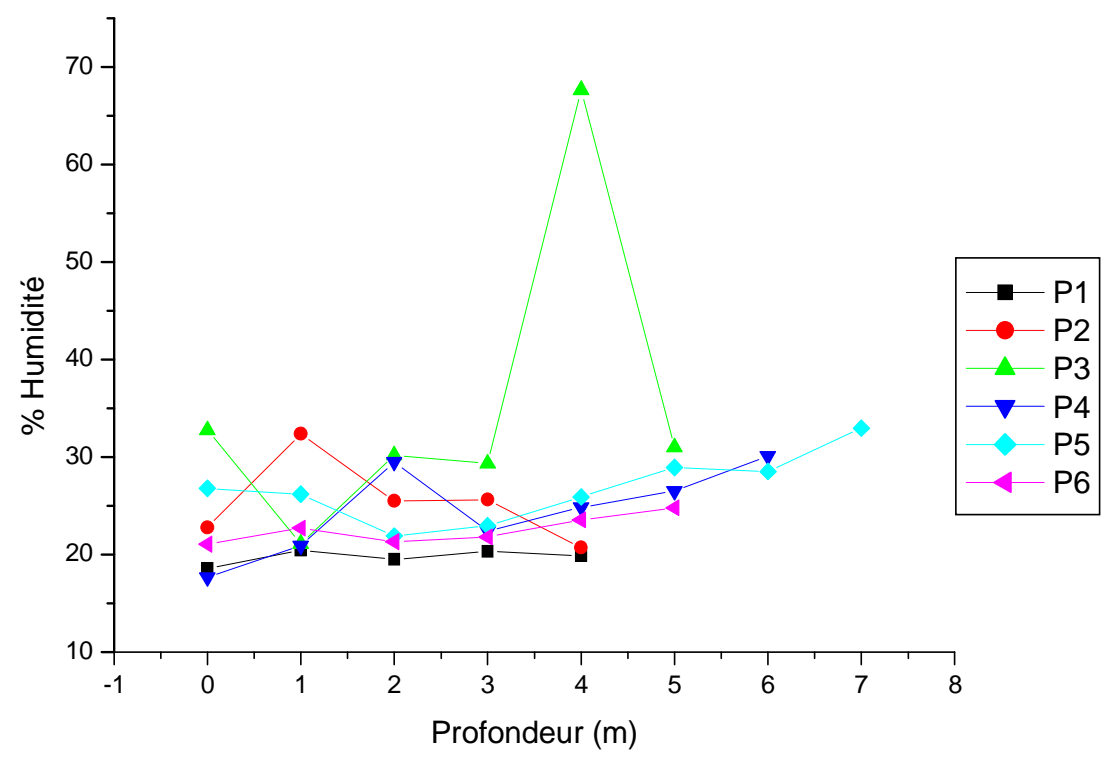

Figure 1: Taux d'humidité des échantillons de déchets des puits en fonction de la profondeur.

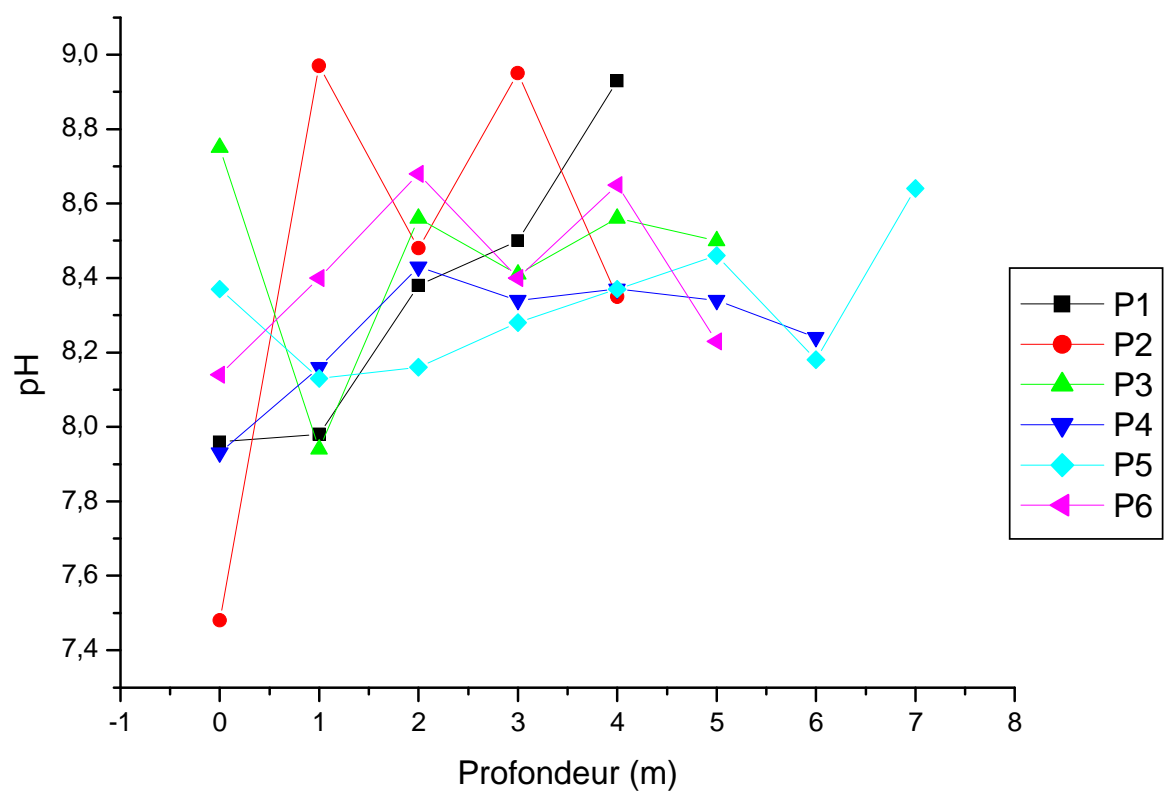

Figure 2 : pH des échantillons de déchets dans les différents niveaux des puits. 


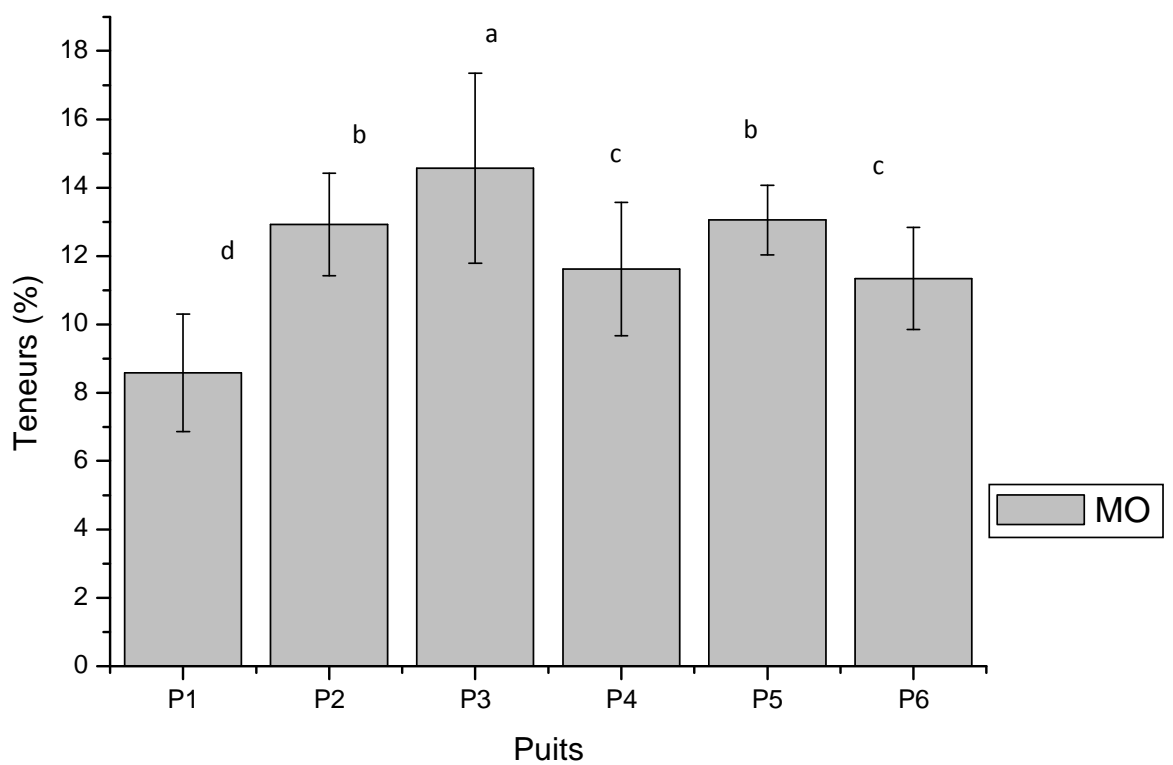

Figure 3 : Teneurs moyennes de la matière organique des déchets des différents puits.

$($ SMD (plus petite différence significative $)=0.89735$. Les moyennes suivies de la même lettre sur les histogrammes ne diffèrent pas significativement entre elles selon le test de Tukey au seuil de 5\%.

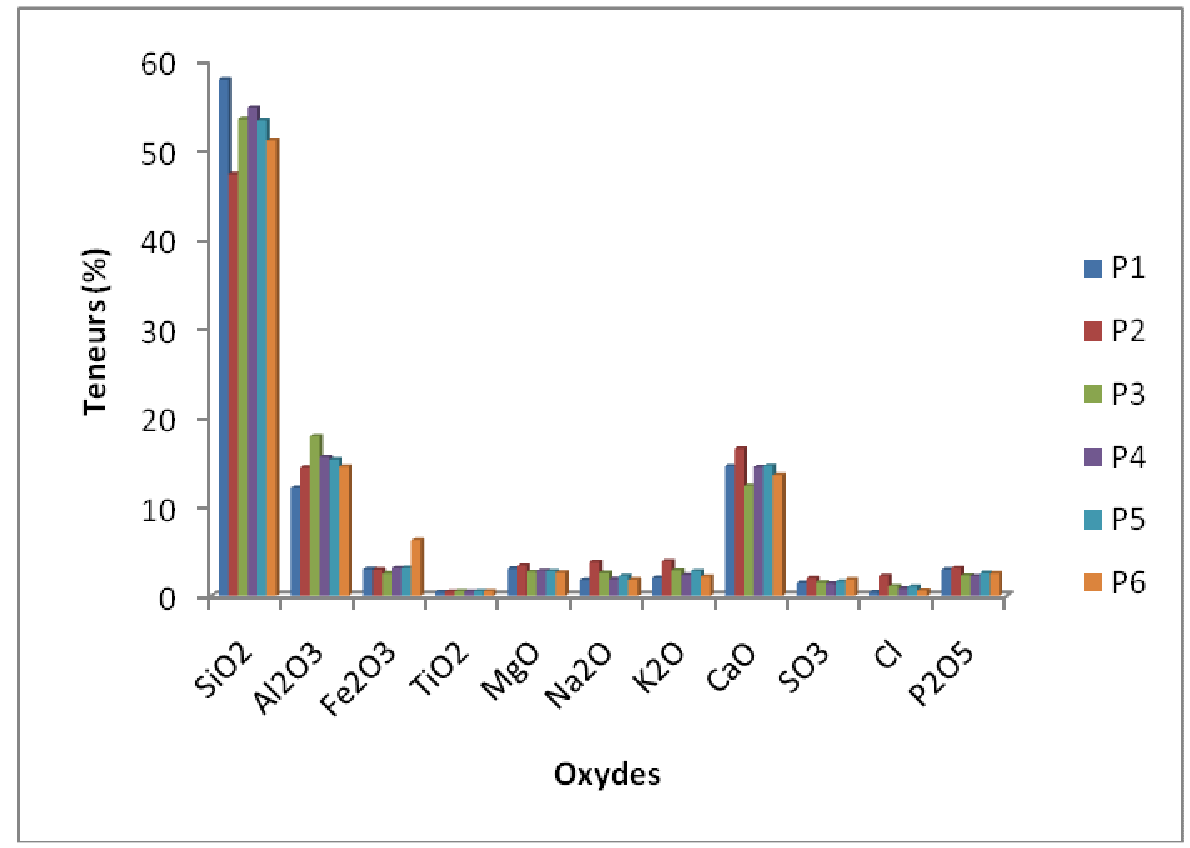

Figure 4 : Composition chimique des échantillons de déchets de la décharge finale 
M. B. BODJONA et al. / Int. J. Biol. Chem. Sci. 6(3): 1368-1380, 2012

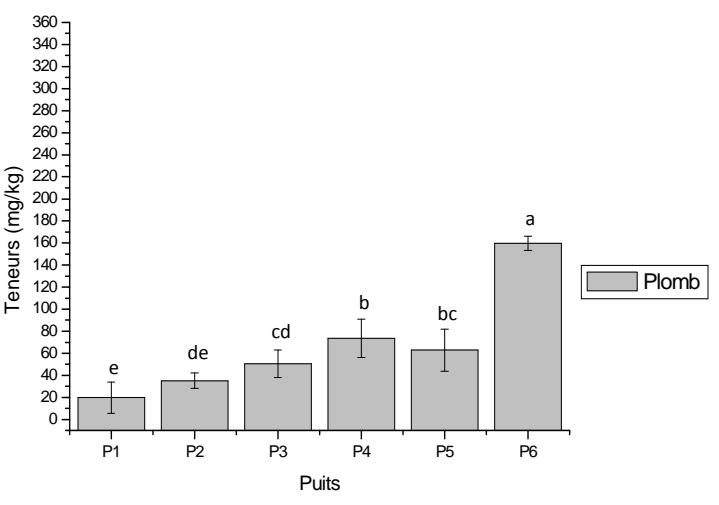

(i) $\quad S M D=15.69661$

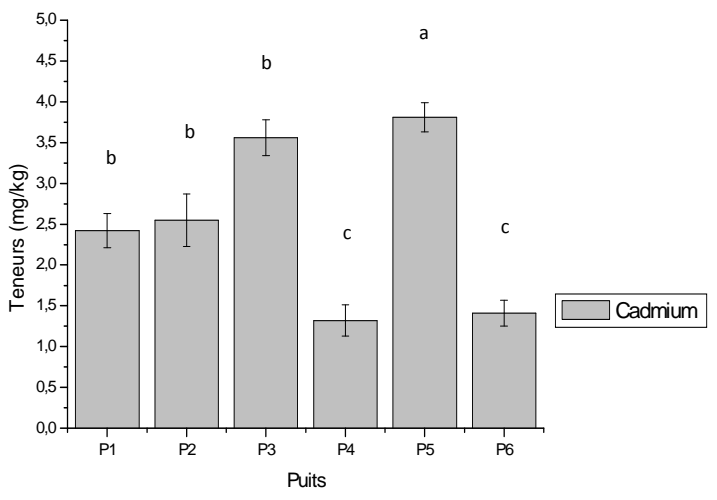

(j) $S M D=0,33496$

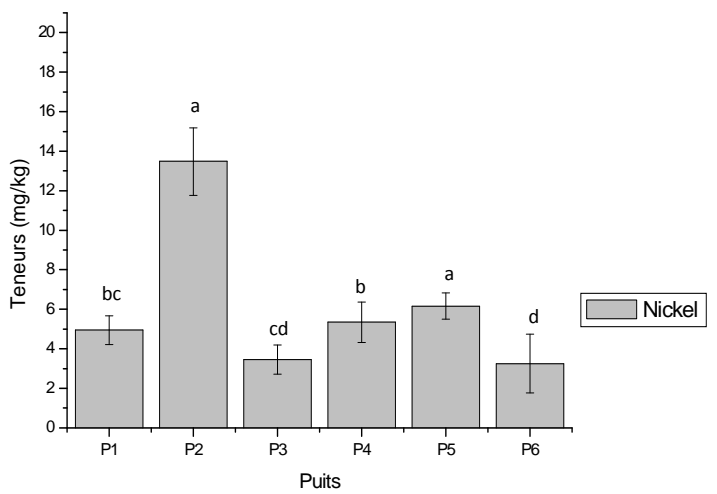

(k)

$S M D=1.56160$ 


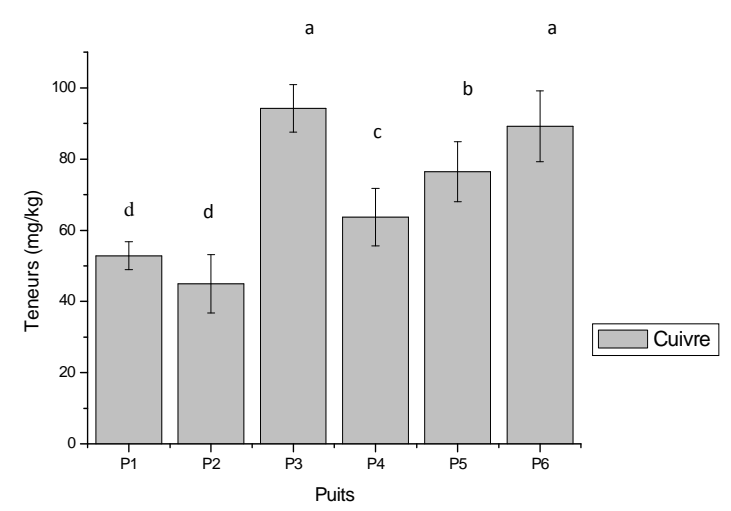

(l) $S M D=9.86623$

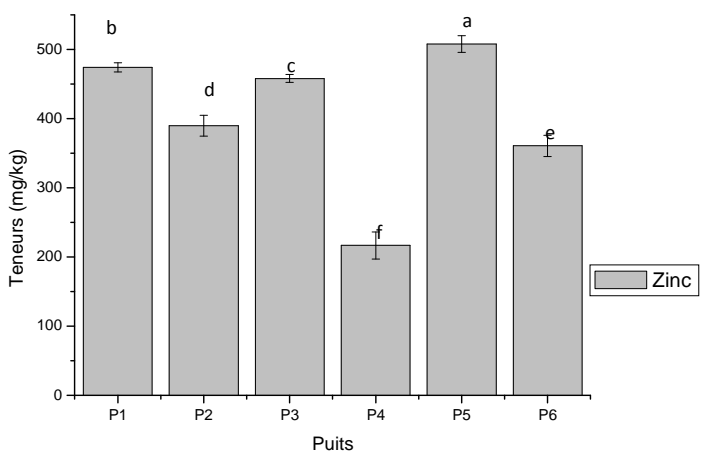

(m) $\quad S M D=15.20343$

Figure 5 : Teneurs moyennes des métaux lourds des déchets des différents puits.

( $S M D=$ plus petite différence significative. Les moyennes suivies de la même lettre sur les histogrammes ne diffèrent pas significativement entre elles selon le test de Tukey au seuil de 5\%.

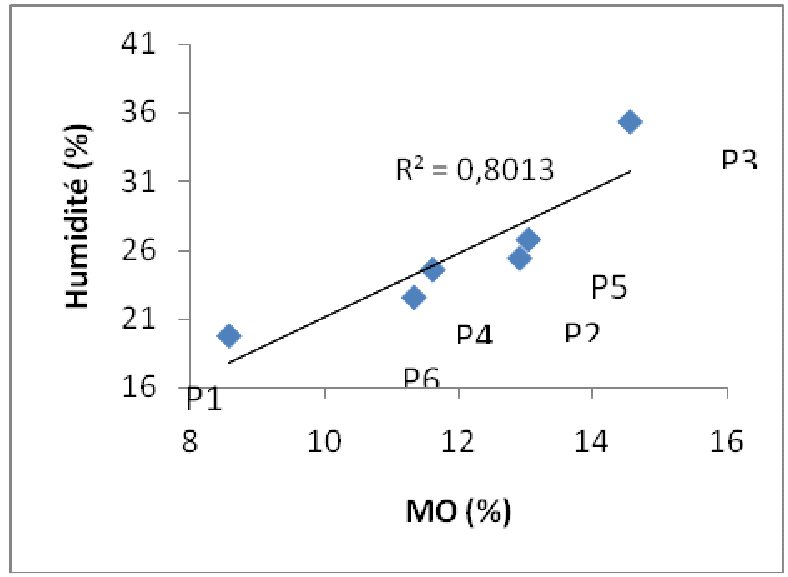

(a) 


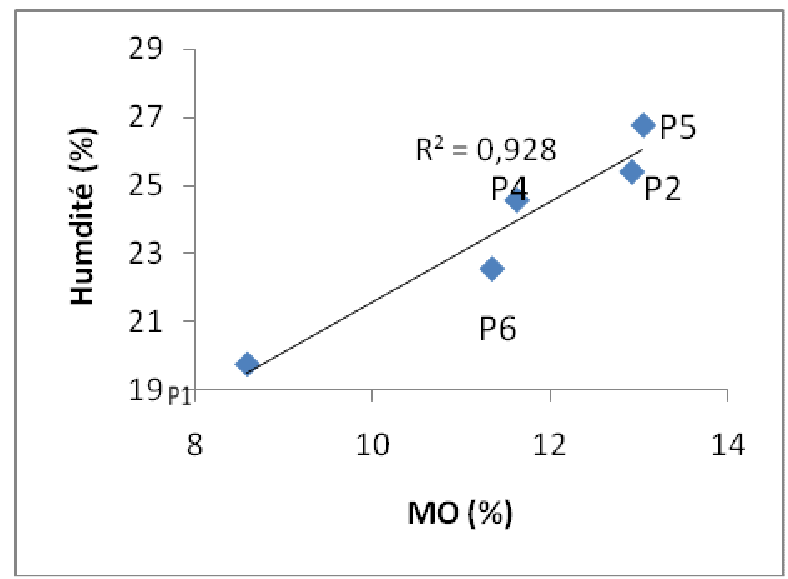

(b)

Figure 6: Corrélation entre l'humidité et la matière organique des échantillons de déchets.

\section{DISCUSSION}

Globalement, on observe que l'humidité des déchets évolue de façon non uniforme au fur et à mesure qu'on descend en profondeur dans le massif de déchets (Figure 1). Cette évolution est due à la nature hétérogène des déchets enfouis à la décharge. La caractérisation physique des déchets de la décharge d'Agoè effectuée par Koledzi et al. (2011), prouve la diversité de ces déchets. Une humidité importante caractérise les déchets des pays en développement (PED), qui sont riches en matière organique (fruits, légumes, reste de nourriture etc.) (Tezanou et al., 2001). Selon Aina (2006), la majorité des déchets des PED ont une teneur en humidité importante comprise entre 50 et $75 \%$. Elle provient de la nature des déchets, constitués en forte proportion de matières putrescibles. Malgré que les prélèvements aient été faits en saison humide, à part les déchets de la profondeur $\mathrm{P}_{3 / 4}$ qui ont un taux d'humidité d'environ $70 \%$, les autres échantillons de déchet ont une teneur inférieure à $50 \%$. La moyenne du taux d'humidité de ces déchets est environ de $25,43 \%$. Cette moyenne est faible comparativement à celle de la littérature estimée entre 30 et $40 \%$ (Kelly, 2002). Cette moyenne est toutefois suffisante pour permettre une dégradation des déchets et de ce fait, une activité des microorganismes.

D'après les résultats, comme l'indique la Figure 2, les pH obtenus sont tous alcalins ( $\mathrm{pH}$ supérieur ou égal à 7). Le $\mathrm{pH}$ alcalin de ces déchets serait dû à la présence de certaines catégories de déchets dans la décharge, comme les fermentescibles, papiers cartons, déchets spéciaux, etc. (Koledzi et al., 2011) qui sont sources de présence de certains éléments alcalins et alcalino-terreux $(\mathrm{Na}, \mathrm{K}$, $\mathrm{Ca}, \mathrm{Mg}$, etc.). Ces éléments, présents sous forme d'ions appelés bases échangeables $\left(\mathrm{Ca}^{2+}, \mathrm{Mg}^{2+}, \mathrm{Na}^{+}\right.$et $\left.\mathrm{K}^{+}\right)$, contribuent à élever le $\mathrm{pH}$ du sol (Martin et al., 1996). Cela peut être dû également à l'hydrolyse bactérienne aérobie ou anaérobie des complexes organiques aboutissant à la formation de sucres simples avec la dégradation de protéines et à la décomposition d'acides organiques dans le massif (Khattabi, 2002; Charnay, 2005). Les carbonates, surtout la calcite $\left(\mathrm{CaCO}_{3}\right)$, assez solubles, et la présence de certains oxydes $\left(\mathrm{MgO}, \mathrm{CaO}, \mathrm{K}_{2} \mathrm{O}\right.$, etc.), ont une influence majeure sur l'élévation du $\mathrm{pH}$ 
des sols (García et al., 2004). Généralement, le $\mathrm{pH}$ acide entraîne la mise en solution des oxydes métalliques et des phases de rétention, la désorption des cations et l'adsorption des anions. Mais le $\mathrm{pH}$ alcalin du massif des déchets de la décharge d'Agoè constitue en sorte un avantage pour l'environnement puisque, l'augmentation de $\mathrm{pH}$ induit souvent la formation d'espèces précipitées qui peuvent limiter la solubilité et la biodisponibilité de toutes les espèces ioniques (Deneux-Mustin et al., 2003).

Une fois déposés dans la décharge, les déchets subissent une dégradation aérobie favorisée par l'oxygène en place (Khattabi, 2002). Les teneurs moyennes des déchets dans les différents puits sont faibles, comparées à celles de la littérature (Figure 3). Puisque, la teneur en matière organique présente dans les déchets d'ordures ménagères est de 59\% (ADEME, 1999). On pouvait s'attendre à un taux de matière organique plus élevé au vue des déchets qui ne sont pas trop vieux (3 ans maximum) et de la proportion des fermentescibles à l'origine estimée à $60 \%$ (Koledzi et al., 2011). Ces faibles teneurs en matière organique sont dues au choix de la fraction effectuée (déchets inférieurs à $10 \mathrm{~mm}$ de diamètre) pour déterminer ce paramètre chimique. Ces faibles teneurs peuvent également être liées à un début de minéralisation de la matière organique. Cette minéralisation est due à la dégradation de la matière organique dans les déchets par l'action des enzymes spécifiques. Ce métabolisme aérobie peut continuer jusqu'à une minéralisation complète des substrats biodégradables et conduit à des métabolites finaux $\left(\mathrm{CO}_{2}, \mathrm{CH}_{4}, \mathrm{H}_{2} \mathrm{O}, \mathrm{CO}_{3}{ }^{2-}, \mathrm{HCO}_{3}{ }^{-}, \mathrm{NO}_{3}{ }^{-}\right.$, $\mathrm{PO}_{4}{ }^{3-}$ et $\mathrm{SO}_{4}{ }^{2-}$ ) (Gordon et al., 1988; Khattabi, 2002).

L'évolution de la décharge est guidée par l'activité microbiologique. Cette activité est tributaire de la teneur en eau de la décharge. Les déchets peuvent demeurer intacts très longtemps à cause de la faible teneur en humidité au sein du massif de déchet (Aina, 2006). De façon générale, les vitesses de dégradation et par conséquent de production de gaz, augmentent lorsque la teneur en eau des déchets crôit (Findikakis et al., 1979; Rees, 1980). Sur la Figure 6a, nous remarquons que le coefficient de corrélation $\mathrm{R}^{2}$ est autour de 0,8013 ; cela montre qu'il y a une corrélation entre l'humidité et la matière organique. Pourtant, le puits P3 est un peu écarté de la courbe de tendance. Cela est probablement dû à l'hétérogénéité des déchets enfouis (Koledzi et al., 2011). Lorsque nous éliminons le Puits 3 (Figure 6b), cette corrélation est d'autant plus parfaite $\left(\mathrm{R}^{2}=\right.$ 0,928). Nous pouvons cependant dire que l'humidité des déchets est corrélée avec la matière organique.

L'analyse à la fluorescence $\mathrm{X}$ des échantillons de déchets montre la présence de plusieurs éléments chimiques. Leur présence peut être expliquée par la diversité des déchets enfouis à la décharge (Koledzi et al., 2011). La forte teneur de l'oxyde de silicium qui est autour de $50 \%$ dans tous les puits, démontre une forte présence du sable dans les échantillons de la décharge. Par ailleurs, la présence des oxydes de magnésium $(\mathrm{MgO})$, de sodium $\left(\mathrm{Na}_{2} \mathrm{O}\right)$, de potassium $\left(\mathrm{K}_{2} \mathrm{O}\right)$ et calcium $(\mathrm{CaO})$ confirme l'hypothèse de $\mathrm{pH}$ alcalin des échantillons évoqués précédemment, puisque ces oxydes favorisent l'élévation du pH d'un milieu (García et al., 2004).

Le plomb est toxique et est l'un des éléments métalliques les moins mobiles du sol. Il est fréquemment retrouvé dans les ordures ménagères des pays en développement à cause de son utilisation comme élément constitutif des piles (Soumaré et al., 2003). Le plomb est retrouvé dans les six puits (Figure 5i). Les teneurs assez élevées du plomb dans certains puits seraient dues à la présence de la matière organique qui n'est pas 
négligeable et à un $\mathrm{pH}$ alcalin pouvant favoriser la rétention des métaux; car un $\mathrm{pH}$ élevé induit souvent la formation d'espèces précipitées qui peuvent limiter la solubilité et la biodisponibilité de toutes les espèces ioniques (Deneux-Mustin et al., 2003). De plus, la présence du plomb dans ces puits n'est pas uniforme. Cela serait dû à la nature hétérogène des déchets (Kelly, 2002 ; Koledzi et al., 2011). La moyenne du plomb des six puits est de $66,91 \mathrm{mg} / \mathrm{kg}$. Elle est inférieure au seuil préconisé par la norme AFNOR NF U 44-041 qui est de $100 \mathrm{mg} / \mathrm{kg}$.

Le cadmium peut être généré par les accumulateurs mais aussi par les plastiques, les verres et les métaux (Bouchakor, 1999). Tout comme pour le cas du plomb, la présence du cadmium dans les puits n'est pas uniforme. Cela serait dû non seulement à l'hétérogénéité des déchets enfouis mais aussi à une dégradation non uniforme de ces déchets (Kelly, 2002). La moyenne totale du cadmium dans les puits estimée à $2,51 \mathrm{mg} / \mathrm{kg}$, est supérieure à la norme AFNOR qui est de 2 $\mathrm{mg} / \mathrm{kg}$. Cette forte teneur du cadmium dans la décharge peut entraîner la pollution du sol et des eaux souterraines, puisque c'est l'un des éléments les plus mobiles (Bouchakor, 1999). De plus, le $\mathrm{pH}$ de relargage du cadmium qui est autour de 6 (Citeau, 2004) n'est pas loin du pH des échantillons de déchet (Figure 2).

Les teneurs $\mathrm{du}$ nickel dans les échantillons sont faibles comparativement à celles de la littérature (Malayeri, 1995 ; Elass et al., 2003). Ces faibles teneurs seraient dues non seulement à la récupération de certains matériaux contenant du nickel pour une éventuelle valorisation (recyclage, réutilisation, etc.), mais du fait que le nickel est un élément très peu retenu par le sol, et migre souvent vers les profondeurs (Elass et al., 2003). La plus forte teneur est trouvée dans le puits P2 (Figure 5k). Les principales sources du nickel dans les ordures ménagères sont: les métaux, les accumulateurs, les colorants utilisés dans le textile et les verres (Lauwerys, 1982 ; Bouchakor, 1999). La moyenne du nickel dans les six puits est de $6,10 \mathrm{mg} / \mathrm{kg}$. Elle est largement inférieure à celle de la norme AFNOR qui est de 50 $\mathrm{mg} / \mathrm{kg}$.

On retrouve de fortes teneurs du cuivre au sein du massif de déchets des puits. Cela est dû à son utilisation dans de nombreuses applications (électronique, chimique, électricité, etc.) (Malayeri, 1995). On note également une distribution non uniforme du cuivre dans les puits de la décharge, liée probablement non seulement à la diversité des déchets mais également à un enfouissement non uniforme. Le pH basique (Figure 2) et une présence non négligeable de la matière organique (Figure 3) des déchets, auraient entraîné une réduction de la mobilité du cuivre vers les profondeurs (Maiz et al., 2000 ; Citeau, 2004). La teneur moyenne du cuivre dans les six puits, qui est de 70,24 mg/kg, est inférieure à la norme AFNOR NF U 44-041 qui est de $100 \mathrm{mg} / \mathrm{kg}$. Le cuivre migre peu, sauf dans les conditions de mauvais drainage ou en milieu très acide (Elass et al., 2003).

Parmi les métaux lourds étudiés, le zinc a de très fortes teneurs dans les sols de la décharge. Ceci est en accord avec Aulin et al., (1997), qui estiment que les concentrations en $\mathrm{Zn}$, sont de 5 à 127 fois plus élevées dans les décharges que dans les sols naturels. Cela est lié à son utilisation compte tenu de son importance dans le monde. Il est plus abondant après le fer (Malayeri, 1995). La présence du zinc dans la décharge n'est pas uniforme. Tout comme pour les autres éléments, on peut lier cela à la nature hétérogène des déchets ou à une dégradation non uniforme des déchets (Kelly, 2002). La moyenne dans les six puits qui est de 401,13 $\mathrm{mg} / \mathrm{kg}$ environ, dépasse largement les seuils préconisés par la norme AFNOR (300 mg/kg). Cette moyenne se situe dans la gamme de la teneur du zinc dans les déchets secs donnée 
par certains auteurs (Flyhammar et al., 1999 ; Alouémine, 2006), entre 380 et $2677 \mathrm{mg} / \mathrm{kg}$.

\section{Conclusion}

Les analyses physico-chimiques effectuées sur les déchets prélevés à la décharge finale ont montré que ces déchets ont une humidité faible avec une moyenne de $25,43 \%$. Les $\mathrm{pH}$ obtenus ont tous un caractère basique avec des teneurs qui varient entre 7,48 et 8,97 . La matière organique de ces déchets est relativement faible et varie entre 8,58 et $14,57 \%$. L'analyse à la fluorescence $\mathrm{X}$ des échantillons de déchets a montré une diversité d'éléments chimiques et une forte présence de sable. Le test de corrélation a montré que l'humidité des déchets est parfaitement corrélée avec la matière organique avec $\mathrm{R}^{2}$ très proche de 1 . Les métaux lourds déterminés $(\mathrm{Pb}, \mathrm{Cd}, \mathrm{Ni}, \mathrm{Cu}$ et $\mathrm{Zn})$ sont présents dans la fraction fine des déchets de tous les puits et leurs teneurs varient en fonction du métal considéré. Comparés à la norme AFNOR NF U 44-041, il ressort que le cadmium et le zinc ont des teneurs supérieures. Ces métaux vont se retrouver complexés avec la matière organique ou piégés sous forme de sulfures métalliques au sein du massif. Il sera alors important de faire d'autres recherches (Spéciation chimique) pour mieux situer l'état de pollution des métaux lourds de la décharge finale d'Agoè.

\section{REFERENCES}

ADEME. 1999. Les Installations de Stockage de Déchets Ménagers et Assimilés: Techniques et Recommandations. ADEME Editions: Paris; 106 p.

Aina MP. 2006. Expertises des centres d'enfouissement techniques de déchets urbains dans les PED: contributions à l'élaboration d'un guide méthodologique à sa validation expérimentale sur sites. Thèse de doctorat $\mathrm{N}^{\circ} 46$, Université de Limoges, p. 2, 59-63.
Aloueimine SO. 2006. Méthodologie de caractérisation des déchets ménagers à Nouakchott (Mauritanie) : contribution à la gestion des déchets et outils d'aide à la décision. Thèse de Doctorat $\mathrm{N}^{\circ} 012$, Université de Limoges, p. 119.

Aulin C, Neretnieks I. 1997. A Material balance for an Industrial Landfill. In Proceeding Sardinia, 5th International Waste Management and Landfill Symposium, Cagliari Italy, 173-180.

Bouchakor F. 1999. Essai d'amendement d'un sol par différents composts d'ordures ménagères et suivi du niveau de contamination par les éléments traces métalliques. Thèse de Doctorat, Université de Tunis II.

Charnay F. 2005. Compostage des déchets urbains dans les PED : Elaboration d'une démarche méthodologique pour une production pérenne de compost. Thèse de Doctorat $\mathrm{N}^{\circ} 56$, Université de Limoges, p. 34, 99-100.

Chofqi A, Younsi A, Lhadi EK, Mania J, Mudry UJ, Veron A. 2004. Environmental impact of an urban landfill on a coastal aquifer (El Jadida, Morocco), Journal of African Earth Sciences, 39(35): 509-516.

Citeau L. 2004. «Etude des colloïdes naturels présents dans les eaux gravitaires de sols contaminés : relation entre nature des colloïdes et réactivité vis-à-vis des métaux ( $\mathrm{Zn}, \mathrm{Cd}, \mathrm{Pb}, \mathrm{Cu})$ ».Thèse de l'Institut National d'Agronomie, ParisGrignon, $236 \mathrm{p}$.

Deneux-Mustin S, Roussel-Debet S, Mustin C, Henner P, Munier-Lamy C, Colle C, Berthelin J, Garnier-Laplace J, Leyval C. 2003. Mobilité et Transfert Racinaire des Eléments en Traces : Influence des Microorganismes du Sol. TEC \& DOC: Paris.

Elass K, Laachach A, Azzi M. 2003. Etude de la biodisponibilité des métaux lourds dans 
les sols agricoles irrigués par des eaux polluées. Revue Francophone d'Ecologie Industrielle, 32: 1-6.

Findikakis AN, Leckie JO. 1979. Numerical simulation of gas flow in sanitary landfills. $J$. of the Environmental Engineering Division, 105(EE5): 927945.

Flyhammar P, Hankansson K. 1999. The release of heavy metals in stabilized Municipal Solid Waste. The Science of the Total Environment, 243-244: 292303.

García MA, Chimenos JM, Fernández AI, Miralles L, Segarra M, Espeill F. 2004. Low-grade $\mathrm{MgO}$ used stabilize heavy metals in highly contaminated soils. Chemosphere, 56: 481-491.

Garcia AJ, Esteban MB, Marquez MC, Ramos P. 2005. Biodegradable municipal solid waste: Characterization and potential use as animal feedstuffs. Waste Management, 25: 780-787.

Gordon AM, Macbride RA, Fisken AJ, Voroney RP. 1988. Effect of landfill leachate spraying on soil respiration and microbial biomass in a northern hardwood forset ecosystem. Waste Mgt. Res., 6: 141-148.

Khattabi. 2002. Intérêts de l'étude des paramètres hydrogéologiques et hydrobiologiques pour la compréhension $\mathrm{du}$ fonctionnement de la station de traitement des lixiviats de la décharge d'ordures ménagères d'Etueffont (Belfort, France). Thèse de Doctorat à l'Institut des Sciences de l'Environnement, 167 p.

Kelly EJ. 2002. Solid waste biodegradation enhancements and the evolution of analytical methods used to predict waste stability. Thesis, Virginia Plytechnic Institute and State University.

Kolédzi KE, Baba G, Feuillade G, Matejka G. 2011. Caractérisation physique des déchets solides urbains à Lomé au Togo, dans la perspective du compostage décentralisé dans les quartiers. Rév. Déchets Sciences et Techniques, 59. (Sous presse).

Lauwerys R. 1982.Toxicologie Industrielle et Intoxications Professionnelles. Masson: Paris.

Lebourg A, Sterkman T, Ciesielski H, Proix N. 1996. Intérêt de différents réactifs d'extraction chimique pour l'evaluation de la biodisponibilité des métaux en traces du sol. Agronomie, 16: 201-215.

M'leyeh A, Srasra E, Cheref A. 2002. Fixation des métaux lourds par les argiles de la décharge municipale de Borj Chekir. Proceedings of International Symposium on Environmental Pollution Control and Waste Management, p. 533546.

Maiz I, Arambarri I, Garcia, Millan E. 2000. Evaluation of heavy metal availability in polluted soils by two sequential extraction procedures using factor analysis. Environmental Pollution, 110: 3-9.

Malayeri EB. 1995. Décontamination des sols contenant des métaux lourds à l'aide de plantes et microorganismes. Thèse de Doctorat, Université de Nancy I.

Martin A, Lamontagne L, Cossette JM. 1996. Etude pédologique de l'Ile Sainte Thérèse, comté de Verchères (Québec).

McLaughlin MJ, Zarcinas BA, Steven DP, Cook N. 2000. Soil testing for heavy metals. Communications in Soil Science and Plant Analysis, 31(11-14): 16611700.

Mokhtaria MM, Eddine BB, Larbi D, Azzedine H, Rabah L. 2007. Caractérisation de la décharge publique de la ville de Tiaret et son impact sur la qualité des eaux souterraines. Courrier $d u$ Savoir, 08: 93-99.

Rees JF. 1980. The Fate of Carbon Compounds in the Landfill Disposal of 
Organic Matter. J. Chem. Tech. Biotechnol., 30: 161-175.

Sbaa M, Chergui H, Melhaoui M, Bouali A. 2001. Tests d'adsorption des métaux lourds $(\mathrm{Cd}, \mathrm{Cu}, \mathrm{Ni}, \mathrm{Pb}, \mathrm{Zn})$ sur des substrats organiques et minéraux de la ville d'Oujda. Actes Inst. Agron. Vet., 21(2): 109-119.

Soumaré M, Tack FMG, Verloo MG. 2003. "Effects of a municipal solid waste compost and mineral fertilization on plant growth in two tropical agricultural soils of Mali. Bioresource Technology, 86: 1520

Tezanou J, Koulidiati J, Proust M, Sourgout M, Goudeau J-C, Kafando P, Rogaume T.
2001. Caractérisation des déchets ménagers de la ville de Ouagadougou (Burkina Faso). Annales de l'Université de Ouagadougou.

Wang QR, Cui YS, Liu XM, Dong YT, Christie P. 2003. Soil contamination and plant uptake of heavy metals at polluted sites in China. J. Environ. Sci. Health Part AToxic/Hazard. Subst. Environ. Eng., 38: 823-838.

Yobouet YA, Adouby K, Trokourey A, Yao B. 2010. Cadmium, Copper, Lead and Zinc speciation in contaminated soils. International Journal of Engineering Science and Technology, 2(5): 802-812. 